\title{
Assessment of interventions in Primary Health Care for improved maternal, new-born and child health in sub- Saharan Africa: A systematic review
}

Lorretta favour Ntoimo ( $\sim$ lorretta.ntoimo@fuoye.edu.ng )

Federal University Oye-Ekiti

Friday Ebhodaghe Okonofua

University of Medical Sciences

Oludamilola Adejumo

University of Medical Sciences

Wilson Imongan

Women's Health and Action Research Centre

Rosemary Ogu

University of Port Harcourt

Seun Anjorin

Women's Health and Action Research Centre

Research article

Keywords: maternal mortality, child mortality pregnant women, primary health care, rural areas

Posted Date: August 19th, 2019

DOl: https://doi.org/10.21203/rs.2.13111/v1

License: (c) (1) This work is licensed under a Creative Commons Attribution 4.0 International License. Read Full License 


\section{Abstract}

Primary health care $(\mathrm{PHC})$ holds great potential to improve maternal, new-born and child health $(\mathrm{MNCH})$ outcomes. Meanwhile, there has been limited documentation of its effect on increasing universal access to maternal, new-born and child health services in sub-Saharan Africa. Also, not adequately known are the most effective interventions to improve the delivery of PHC services in the region. We conducted a systematic review of empirical evidence and interventions at the primary health care level for effective delivery of MNCH care in subSaharan Africa. Using terms related to primary health care and $\mathrm{MNCH}$, we searched African Journals Online (AJOL), PubMed/Medline, Popline, ScienceDirect, Google Scholar, WHO Repository (IRIS), Directory of Open Access Journals (DOAJ), Cochrane Library and reference lists for studies published in English between 2000 and 2017. Studies were included in the search if they reported interventions, and strategies implemented to improve quality and access to primary health care for maternal, new-born and child health in sub-Saharan Africa. A total of 25 studies were included in the review. Effective interventions included financial incentives, task-shifting, community-directed engagements, training of providers, mobile health, cost-sharing and supportive supervision among others. The results documented in these studies indicate that effective delivery of primary health care will significantly improve maternal, new-born and child health in sub-Saharan Africa. However, strategies to scale and sustain the successes need to be in place. The protocol was registered on Prospero (Registration number CRD42019126029).

\section{Introduction}

The high rate of maternal, neonatal and child mortality continues to be a major public health concern in many sub-Saharan African countries [1, 2]. For instance, the highest estimated maternal mortality ratio (MMR) worldwide is in Sierra Leone (1360/100000 live births), and 18 other countries in sub-Saharan Africa are estimated to have very high MMR ranging from 500 to 999 [3]. Weak health systems made worse by conflicts, disasters, and forced displacement among other factors in the region increases exposure to the risk of death for mothers and children. Many of these deaths occur in hard-to-reach and underserved rural communities in the region, especially in illiterate and poorly educated women $[4,5]$. It is increasingly evident that poor access to quality health care by socially disadvantaged women is at the root of the high rate of maternal and child mortality in many parts of sub-Saharan Africa. Evidence from Demographic and Health Surveys in sub-Saharan African countries suggests that a high proportion of pregnant women who have no formal education and those with only primary-level education deliver with unskilled traditional birth attendants rather than with skilled attendants in the formal health system, exposing them to higher risks of maternal and neonatal morbidity and mortality [6].

To address this, the World Health Organization has recommended Primary Health Care (PHC) as capable of increasing women's access to the level of care needed to attend to the majority of the minor conditions that lead to avoidable maternal, new-born and child morbidity and mortality [7]. Primary health care is a form of accessible, affordable and effective health care that enables citizens to enter the formal health system to receive evidencebased promotive, preventive and curative care necessary to avert morbidity and mortality. It includes at least the education of women relating to prevailing health problems and the methods of preventing and controlling them; promotion of food supply and proper nutrition; an adequate supply of safe water and basic sanitation; maternal and child health care, including family planning; immunization against the major infectious diseases; prevention and control of locally endemic diseases; appropriate treatment of common diseases and injuries; and the provision of essential drugs. Consequently, most countries in sub-Saharan Africa have adopted PHC as the 
foundation of their health care systems in efforts to reach the most vulnerable persons [8]. A country like Ethiopia has made impressive progress in providing primary health care across the country, particularly to rural communities through an elaborate health extension programme with notable impact on maternal and child health $[9,10]$. In the Gambia, Hill et. al [11] documented evidence of significant decline in childhood mortality in communities with a primary health care facility compared to communities without a PHC in the 1980s. However, the gain in childhood mortality in the Gambia waned when PHC programme began to receive reduced governmental support after 1994. Nigeria recently revitalized its PHC approach by recommending the creation of Primary Health Care Development Agencies in its 36 States and identifying nine indicators to measure their successful performances $[12,13]$. The policy referred to as "Primary Health Care Under One Roof (PHCUOR)", if well implemented promises to strongly position PHC for effective delivery of maternal and child health care to under-served communities in Nigeria.

However, despite the promise of $\mathrm{PHC}$, there has been limited documentation of its effectiveness in improving maternal, new-born and child health in sub-Saharan Africa, a region with the highest burden of maternal and child mortality and morbidity. Also, not adequately known are the best approaches for developing and implementing policies on PHC to enable them respond to the health needs of women and children. However, a few systematic reviews have been conducted on best practices in effective PHC delivery in sub-Saharan Africa but they are limited in focus. For instance, Christopher et al [14] reviewed studies on the effectiveness of Community Health Workers in sub-Saharan Africa. Their study was limited in its coverage, focusing only on child health and on three countries in the region (Gambia, Ghana and Benin).

Therefore, through a review of the existing literature, we specifically focused on how effective primary health care has been positioned using different interventions to improve maternal, new-born and child health care, including family planning and the prevention of related morbidity and mortality in sub-Saharan Africa. We solicited information on community, outreach and facility-based interventions, strategies and approaches that have been applied to improve access and quality of services for $\mathrm{MNCH}$ at the primary health care level. We believe this review of existing studies will be useful for developing policies and programmes for improving the quality of delivery of primary maternal and child health services in the region.

\section{Methods}

\section{Search Strategy}

The Preferred Reporting Items for Systematic Review and Meta-Analysis (PRISMA) statement was followed in conducting this review and the protocol was registered on Prospero (Registration number CRD42019126029). The search for literature targeted peer reviewed and published journal articles. We searched bibliographic databases including African Journals Online (AJOL), PubMed/Medline, Popline, ScienceDirect, Google Scholar, WHO Repository (IRIS), Directory of Open Access Journals (DOAJ), and Cochrane Library. Also, searched were reference lists of relevant systematic reviews and other articles. Keywords used in the systematic search of the literature included key words drawn from the WHO recommended interventions for pregnancy, childbirth, postpartum, newborn, infant and child care and family planning at the community and first-level facility [2]. Also included were synonyms and Medical subject Headings (MeSH) of each database (Medline/PubMed). 


\section{Inclusion Criteria}

The evidence for this review was drawn from intervention studies that were PHC facility-based, community-based and related to the functions of a PHC, with outcomes designed to improve quality and access to PHC services for maternal, new-born and child health care. Quality of service was defined in terms of improvement in self-reported or otherwise tested competence of providers in providing PHC functions for $\mathrm{MNCH}$. Access was defined as improvement in utilization of PHC facility, community and outreach services by women for $\mathrm{MNCH}$. Studies were included if they used quantitative design, were written in English, conducted in sub-Saharan Africa between 2000 and 2018 and published in a peer-reviewed journal. The review was limited to 2000 and 2018 in order to assess the progress made in sub-Saharan Africa in achieving health for all through primary health care before the Alma Ata deadline in 2000 and efforts made toward the attainment of the Millennium Development Goals. The initial search using the various terms generated 2055 articles (Figure 1). After removing duplicates, 119 potentially relevant articles were retrieved for full-text review and 25 met our inclusion criteria. Articles were retrieved and screened by two authors (LFCN and SA).

\section{Data extraction, synthesis and analysis}

Using a data extraction form we extracted the following information from each study: authors, date of publication, setting, data source, research design, intervention/strategy implemented, follow-up period, desired outcome measured, and major findings. The information extracted from the studies were qualitatively analysed and organized into thematic areas on types of interventions or strategies for improved quality of care and utilization of primary health care services. Improvement in quality and access to $\mathrm{PHC}$ for $\mathrm{MNCH}$ care was our primary outcome of interest. Thus, interventions, strategies and approaches were assessed to be effective if the reported outcome was statistically significant, resulted in quantified or self-reported improvement in quality of care provided and in utilization of services for $\mathrm{MNCH}$ care.

An assessment of the quality of evidence was conducted using the Grading of Recommendations Assessment, Development and Evaluation (GRADE) approach $[15,16]$. Quality of evidence was classified into high, moderate, low. In the GRADE approach high quality means that further research is very unlikely to change the confidence in the estimate of effect; Moderate quality indicates further research is likely to have an important impact on our confidence in the estimate of effect and may change the estimate Low quality-Further research is very likely to have an important impact on our confidence in the estimate of effect and is likely to change the estimate Studies were ranked on a scale of 3-1 ( $3=$ high quality, $2=$ moderate quality and 1 is low quality) on eight criteria: Study design, adequate description of the intervention, adequate description of the outcome measures before and after intervention, period between the intervention and outcome more than one year, analysis involved inferential statistics and control of potential confounders, results clearly reported, reported positive results, and limitations/biases reported. The highest obtainable score was 24 . The cumulative quality score of each study was converted to percentage. Scores $\geq 70 \%$ was classified as high quality (3), medium quality score was from $50 \%$ to $<70 \%$ (2), and low-quality score if $<50 \%$ (1). With respect to study design, randomized control trials were rated as high quality except where there are inconsistencies in the results, and reporting bias, case-control studies, pre-post or before and after without control, were rated moderate, cross sectional and descriptive studies were rated low. Summary of the reviewed literature is presented in Table 1. 


\section{Fig 1: Flow diagram of study selection according to PRISMA Flow Chart}

\section{Results}

The included studies differed in terms of study design, method of data collection, type of data collected and analytical strategies. Thus, the articles were grouped according to similar interventions for narrative synthesis.

\section{Characteristics of the included studies}

The included articles are relatively a good representation of studies across sub-Saharan Africa as the studies covered all the regions except Central Africa. Studies from Eastern Africa were the highest $(n=12)$ : Tanzania [1722], Rwanda [23, 24], Ethiopia [25, 26], Kenya [27] and Uganda [28]. There were five studies from Southern Africa: South Africa [29-31], Malawi [32], and Zimbabwe[33]. Studies conducted in Western Africa were 9: Nigeria [24, 34-38], Burkina Faso [39], Senegal [40], and Ghana [41]. The majority of the studies used quasi-experimental designs ( $n=16)$; seven studies were cluster/community randomized trials, and three were prospective studies that engaged descriptive and mixed methods. The subject in three studies was financial incentives in the form of results-based payments, conditional and unconditional cash transfers. One study evaluated the effect of increasing the number of facility-based providers; another one study evaluated task-shifting. Community-directed interventions were the subject of 10 studies, and in some cases the community-based interventions were implemented with improvements in facility-based activities. In three studies, interventions were focused on upgrading the skills of facility-based providers but in one of the three studies, a community-based intervention was also implemented alongside providers' training. Other themes were mobile health $(n=3)$, cost-sharing $(n=1)$, introduction of a sub-specialty $(n=1)$ and supportive supervision $(n=3)$. Assessment of the quality of evidence showed that 15 studies were of high quality ( $\geq 70 \%)$, particularly the randomised control trials, 11 were assessed moderate in quality of evidence and none was of low quality.

\section{Interventions and their effect on maternal, new-born and child health}

Included in this review were studies that examined facility and community-based interventions and strategies implemented to improve the quality of care and utilization of primary health care services for maternal, new-born and child health in sub-Saharan Africa. The results are presented qualitatively using themes that describe the types of intervention. The interventions and strategies and their outcomes were described under each theme.

\section{Financial Intervention}

Financial incentives in forms of payment for performance and conditional and unconditional cash transfers to households and women increased the quality of care and access to primary health care facilities for $\mathrm{MNCH}$ preventive and curative services, albeit there were variations across the different outcome indicators. For instance, in a prospective study conducted by Basinga et al [22] the potential of a payment for performance scheme to increase the use and quality of $\mathrm{MNCH}$ services was assessed using antenatal care visits and institutional 
deliveries, quality of antenatal care, and child preventive care visits and immunization as outcome measures. Facilities in the intervention group experienced a $23 \%$ increase in the number of institutional deliveries and $56 \%$ increase in the number of preventive care visits by children aged 23 months or younger and $132 \%$ rise in visits for children aged between 24 months and 59 months $(132 \%)$ and a significant increase in antenatal care quality (0.157 standard deviations $-95 \% \mathrm{Cl} 0 \cdot 026-0 \cdot 289)$. Although antenatal quality reportedly increased there was no improvement in the number of women who completed four or more antenatal visits and the number of children who received full immunization. In Zimbabwe [33], conditional cash transfer (CCT) and unconditional cash transfer (UCT) was associated with better outcome in the intervention sites than the control clusters where no cash transfer was implemented. Compared with the control group, the number of children aged $0-4$ with birth certificates increased by $16.4 \%(95 \% \mathrm{Cl} 7.8-25.0)$ in the CCT group and $1.5 \%(95 \% \mathrm{Cl}-7.1-10.1)$ in the UCT group, but the difference was only significant in the CCT group. For complete vaccination of children aged $0-4$, there was an increase by $3.1 \%$ in the UCT group and $1.8 \%$ in the CCT group but there was no difference with the control group. Similar to Zimbabwe, conditional cash transfer intervention in a pilot study conducted in 37 PHC facilities in 9 Nigerian States improved maternal health. Compared to the control sites, conditional cash transfer was associated with a statistically significant increase in the monthly average number of women who completed 4 or more antenatal care visits by 15.11 visits per 100,000 catchment population ( $95 \% \mathrm{Cl}: 7.38-22.85)$, and the number of women receiving two or more tetanus toxoid doses during pregnancy by 21.66 cases per 100,000 catchment population (95\% Cl:9.23-34.08) [34].

Cost-sharing in the form of Community Health Fund (CHF) was another form of financial intervention.Murshi [17] assessed the impact in Tanzania on quality of care, attendance and utilization of PHC services as the CHF improves The program presented two options of paying medical bills to households-over the counter cash payment on receipt of services or pre-payment through the CHF. Under the CHF, participating households pay a fixed amount per year to cover their medical expenses in public PHC facilities within the district. All the revenues are kept in a common district account that is managed by the District Medical Officer. The government increased regular inflow of medical supplies to PHC facilities. With increased regular supply, revenue from the CHF was used

mainly for minor renovations, extensions of health facility premises, and occasional purchase of medical supplies when there is a shortage. The programme resulted in improvement in the quality of primary health care services, attendance by all categories of clients for $\mathrm{MCH}$ care almost doubled by year 2005 , and under -5 mortality declined in one district.

\section{Human Resource Intervention}

One of the challenges of effective functioning of PHC in sub-Saharan Africa is the dearth of skilled health providers. Interventions addressing this barrier revolved around increasing the number of nurses/midwives, community health workers (CHWs), task shifting and recruitment of community health volunteers with or without the assistance and involvement of the target communities $[18,24,25,27,34]$. The volunteers are trained in diverse target components of $\mathrm{MNCH}$ for outreach to individuals, families, households, small groups, and communities. In a few settings they received stipend, and in many cases, they were not given any stipend. In Kenya the volunteers were trained to deliver reproductive health messages one-on-one and with small groups and they received no stipends. The effect of the human resource intervention in Kenya was a significant increase in knowledge of maternal and child health and facility delivery for women who were exposed to the health message [27]. In a Nigerian study [34], recruitment of more midwives between 2012 and 2014 reportedly resulted in a $42 \%$ increase in 
new antenatal care visits, $56 \%$ in skilled birth attendance, $33 \%$ in postnatal visits and $66 \%$ in use of contraceptives. The recruitment of young local women as Health Extension workers and the "model family" strategy in Ethiopia [25] was associated with increased programme intensity which increased the odds of antenatal care (OR $1.13 \mathrm{Cl}: 1.03-1.23)$, receiving iron supplement (OR $1.14 \mathrm{Cl}: 1.02-1.26)$, receiving at least 2 Tetanus Toxoid injections (OR $1.09 \mathrm{Cl}$ : 1.00-1.18), birth preparedness (OR 1.31 Cl: 1.19-1.44), postnatal care (OR $1.60 \mathrm{Cl} 1.34-1.91)$ and initiation of breastfeeding immediately after birth (OR $1.10 \mathrm{Cl}: 1.02-1.20)$, knowledge of danger sign during childbirth ( $\beta=0.06 \mathrm{Cl}: 0.02-0.10)$, postnatal period ( $\beta=0.04 \mathrm{Cl}$ : $0.00-0.07)$ and neonatal danger sign ( $\beta=0.04 \mathrm{Cl}$ : 0.00-0.07). In Tanzania [18], the use of safe motherhood promoters (men and women) who were married, literate and accepted by the community resulted in a significant improvement in skilled deliveries (34\% to $51.4 \% p<0.05)$, early booking for antenatal care $(18 \%-56.9 \% p<0.01)$ and four or more antenatal care visits $(42.2 \%-51.3 \%)$ for primigravid mothers. Increasing the number of professionally trained providers in a PHC facility as in the Nigerian study or trained volunteers as in Kenya, Ethiopia and Tanzania who provide community-based PHC services is germane for improved quality and access to PHC services for $\mathrm{MNCH}$.

Another component of human resource intervention was capacity-building through training and retraining of providers. In a community-directed intervention in Nigeria ([24], PHC staff were trained on malaria and malaria in pregnancy and they reached out to community leaders and volunteers. The program significantly increased the coverage of intermittent prevention treatment (IPTp) and insecticide-treated nets and prenatal care attendance in the intervention communities compared to the control communities. The proportion of pregnant women taking at least two sulfadoxine-pyrimethamine doses during pregnancy was five times in the experiment communities compared with three times in the control group $(P<0.001)$. in another Nigerian study, a two-day training in ten $\mathrm{PHCs}$ on child feeding resulted in improved health providers' knowledge, attitude and practices of one-on-one individualized infant and young child feeding counselling in line with global Infant and Young Child Feeding recommendations [36]. A capacity building intervention in KwaZulu-Natal, South Africa [30] with a focus on using routine data for problem identification, target setting and monitoring for HIV, PMTCT, maternal and child health $(\mathrm{MCH})$ increased the coverage of CD4 testing from 40 to 97\%; uptake of maternal nevirapine increased from 57 to $96 \%$; uptake of infant nevirapine increased from 15 to $68 \%$; while six weeks PCR testing increased from 24 to $68 \%$.

Noteworthy is that although the human resource interventions were primarily aimed to improve maternal and newborn health, the capacity of the providers (professionals and volunteers) are also built as an intended or unintended consequence. For instance, In Ethiopia, a home-visit package reportedly improved the capacity and confidence of the home-visit team of Health Extension Workers, Community Health Development Agents, and Traditional Births Attendants to provide better maternal and new-born care [26]. In Tanzania, 94\% of the SMPs reported that the training was useful to themselves and their work [18].

\section{Home-visits Intervention}

Some of the studies reported specialised packages of home-visits to increase utilization of PHCs services for maternal, new-born and child care $[21,26,31]$. The specialized home visits were particularly useful for recognition of danger signs by mothers, improvement in child health, male involvement, and adequate antenatal and postnatal care. In Kwazulu-Natal, South Africa, CHWs delivered a package of home visit, Good Start Saving Newborn Lives, which involved provision of essential maternal and new-born care to pregnant and postnatal women and their new-born to prevent mother to child transmission of HIV. Mothers were counselled and referred to a PHC 
where necessary. Referrals to PHC clinics increased and compliance to the referrals was reported to be $95 \%$ among mothers who completed the referral forms. Also, compared to none in mothers who did not complete the referral forms, $51 \%$ of mothers who completed the referral forms recognised danger signs and reported improved infant health [31]. Specialised visits by CHW who were trained in Home-Based Life-Saving Skills (HBLSS), to visit identified pregnant women and their husbands four times to provide education in two districts of Tanzania [21] was associated with a statistically significant improvement in male involvement in the intervention sites (39.2\% $80.9 \% \mathrm{Cl}: 28.5-53.8)$. In the intervention sites, there was a statistically significant net intervention effect (NIE) in women's knowledge of at least three danger signs during pregnancy (NIE 21.3\%, 95\% Cl: 13.7-28.9), childbirth (NIE 13.9\%, 95\% Cl: 10.5-17.4) and postpartum (NIE 15.1\%, 95\% Cl: 9.2-21); proportion of men who accompanied their wives to antenatal care visits (NIE 16.4\%, Cl:5.6-27.2), childbirth (NIE 33.1\%, 95\% Cl: 24.142.1), and joint decision between wife and husband about place of delivery (NIE 38.5\%, 95\% Cl: 28.0-49.1). The proportion of men who mentioned at least three danger signs during pregnancy, childbirth and postpartum significantly improved in the intervention relative to the control sites (NIE $27 \%, 95 \% \mathrm{Cl}: 15.3-38.5$ ), took at least three birth preparation and complications readiness actions (NIE $26.8 \%, 95 \% \mathrm{Cl}: 15.3-38.2$ ). In Ethiopia, the home visit strategy was conducted by a team comprising a Health Extension Worker, Community Health Development Agent and Traditional Birth Attendant trained in HBLSS who taught skills to women and their care givers in their second and third trimester of pregnancy. Behaviour change communication tools such as films and drama among others were also used. This intervention significantly increased the number of women who received 4 or more antenatal care visits, use of skilled providers for childbirth, and postnatal care for mothers and new-borns.

\section{Birth preparedness and complications readiness Intervention}

Birth preparedness plan between a provider and a pregnant woman was reported in only one study conducted in Tanzania and was associated with increase in skilled delivery, uptake and early initiation of postnatal care. In Ngorongoro district, Arusha region, Tanzania, the intervention consisted of the introduction and promotion of birth plans during antenatal care visit to prepare women and their families for birth and complication readiness was implemented [22].. Health providers at the intervention sites were given a birth plan implementation guide and instructions on how to assist women develop a birth plan. They discussed with women on planned place of delivery, the importance of skilled delivery care, transport arrangement to the health facility during delivery or an emergency, funding arrangements for delivery or emergency, identification of possible blood donors, identification of a birth companion if desired and appropriate, and support in looking after the household while the woman is at the facility. Also discussed were strategies for accessing skilled care, recognition of danger signs during pregnancy, labour and postpartum. Women were asked about their choice of place of delivery (dispensary or hospital) and with her consent, their male partners or any other persons they identified as a carer were invited for subsequent discussion. The birth plans were written, one copy for the woman and a second copy retained at the dispensary. Relative to the control sites, skilled delivery care was $16.8 \%$, higher in the intervention sites $[95 \% \mathrm{Cl}$ 2.6-31.0; $p=0.02$ ]postnatal care utilisation in the first month of delivery was higher (difference in proportions: $30.0 \%$ [95\% Cl 11.3-46.7; $p<0.01]$ ) and was also initiated earlier (mean duration $6.6 \pm 1.7$ days vs. $20.9 \pm 4.4$ days at the control, $\mathrm{P}<0.01$ ).

\section{Mobile Health (mHealth) Intervention}


Mobile health was shown to facilitate emergency medical responses, point-of-care support, improvement in access and adoption of maternal, prenatal and neonatal service.. An assessment of the impact of using mHealth (Chipatala cha pa Foni) in Malawi [32] showed a large, positive effect of the project on the aggregate home-based care for child health $(p<0.01)$, and a sharp, negative impact on facility-based care seeking for fever among children whose mothers/caretakers used the services offered by the intervention. A cluster randomized controlled trial conducted in primary health care facilities in Zanzibar, Tanzania demonstrated that an intervention using mobile phones with a voucher component for the wired women resulted in a $50 \%$ significant reduction in perinatal mortality (odds ratio $0.50,95 \% \mathrm{Cl} 0.27-0.93$ ) in the intervention clusters. The mobile phone intervention was associated with increase in uptake of four and more antenatal care visits. In the intervention group, $44 \%$ of the women received four or more antenatal care visits versus $31 \%$ in the control group (OR, 2.39; $95 \% \mathrm{Cl}, 1.03-5.55)$ $[19,20]$.

\section{Introduction of a sub-specialty}

The intervention reported in Cox et al [29] was the introduction of sub-specialty service (paediatric surgical clinic) at the primary health care level in Western Cape, South Africa. Over a 58-month period, 1, 171 children aged 0-19 were seen, the largest group being under one year. The correct diagnosis was established by the nurse practitioners in 255 children (71\%). In total, 597 patients were referred directly to an appropriate care facility, while 574 patients could be managed entirely at the clinic level. The clinic allowed for timely surgical intervention in $65 \%$ of surgical cases, thereby decreasing inappropriate tertiary referrals. The introduction of a subspecialty in primary health care facilities in Western Cape, South Africa improved accuracy in diagnosis and appropriate diagnosis and highlights the preventative and cost-effective role of a surgical clinic at primary health care level.

\section{Supportive supervision Intervention}

Contrary to the top-down supervision approach which tends to concentrate more on administrative functions and emphasize fault-finding, supportive supervision emphasizes mentoring, joint problem-solving and two-way communication between the supervisor and supervisee. A significant improvement in the quality and access to sexual and reproductive health services was reported after implementing supportive supervision in different settings. In Senegal, Suh, Moreira \& Ly [40] reported improvement in technical competence in infection prevention improved by $28 \%$ and $32 \%$ in Theies and Louga, respectively. Skills in family planning consultation improved by $16 \%$ in Louga region and 10\% in Tivaoune. The management of staff and services, record-keeping, and community involvement also improved considerably.

la facility-based intervention to measure the effect of supportive supervision of PHC workers in malaria case management for under-5 children was implemented in Jos, Nigeria Bello et al [38]. Using the WHO guidelines, training in supportive supervision was conducted in the intervention sites (PHCs) while the traditional supervisory method continued in the control sites. The mean score on knowledge of malaria significantly increased from preintervention score of $10.3 \pm 1.4$ to $113 \pm 1.5$ post intervention, whereas it decreased in the control group from $10.6 \pm 1.7$ to $10.5 \pm 2.3(t=3.57 p<0.0015)$. Malaria management practices mean score for the intervention group increased from $5.8 \pm 1.7$ before intervention to $7.1 \pm 6.4$ post-intervention. Scores for the control group decreased from $6.2 \pm 1.7$ pre-intervention to $5.7 \pm 1.6$ post-intervention; but the difference was not statistically significant. Statistically significant increase in the percentage of workers who would refer to a secondary facility following a 
poor response to treatment increased from $47.3 \%$ to $84.3 \%$ post-intervention for the experiment group. In Uganda, after one year of the intervention significantly more functioning tippy taps $(p<0.002)$ were present in the intervention villages ( $47 \%$ ) than in control villages (35\%). Visits to pregnant women by CHVs increased from $2 \%$ of pregnant women visited once before the study to $33 \%$ of pregnant women visits 2.3 times during the study for the control sites. At the intervention, the visits increased from $9 \%$ once pre-intervention to $46 \%$. Visits to new-borns improved from $28 \%$ once visit to $44 \% 1.8$ time visit at the control sites; at the intervention sites, it increased from $9 \%$ once visit to $67 \%$ an average of 2 visits [28].

\section{Combined interventions}

In some of the studies, more than one strategy was employed to achieve the expected outcomes [37, 39, 41]. For instance, to increase delivery in a primary health care facility, a community education programme for traditional rulers, women groups, religious organisations and traditional birth attendants was implemented in Port-Harcourt, Nigeria. In addition, the delivery fee was collected upfront with antenatal care fee at the time of registration for antenatal care. The amount was equivalent to the average charge by TBAs in the community, and less that the fee for normal delivery in a teaching hospital. The effect was assessed one year after. Antenatal registration

increased by $15.04 \%$ after the programme. The ANC-Delivery ratio of the health centre increased by $3.09 \%$, p-value $>0.05$ [37]. In Burkina Faso, Brazier et al [39] reported a combination of facility and community-based interventions. The intervention package at the facilities (primarily focused on primary health centres) included activities to improve the quality, availability, and accessibility of routine and emergency obstetric care (EMOC): upgrading the skills of all maternity care providers through training in routine and emergency obstetric care; addressing gaps in essential obstetric equipment and supplies; strengthening the referral system; introducing a quality assurance methodology for maternal health services; and improving management systems. Communitylevel interventions focused on increasing women's information about services. There was a large increase in the per cent of births in health facilities in the intervention district from $29 \%$ at baseline to $57 \%$ at end line (Chi2 = 185.3, $p<0.001)$; increase in delivery by skilled attendants from $24 \%$ to $56 \%$ (Chi2 $=256.4, p<0.001)$. In the comparison district, there was no significant increase in number of births at a health facility between the baseline and end line, but there was a slight increase in the proportion of births assisted by a skilled attendant from $32 \%$ to $36 \%($ Chi2 $=6.2, p<005)$.

\section{Discussion}

The objective of this review was to document the interventions aimed at addressing the improvement of PHC service delivery for reducing maternal, new-born and child mortality in sub-Saharan Africa. Specific evidence was sought for interventions, strategies and approaches that have been implemented to improve quality and access to primary health care for maternal, new-born and child health in sub-Saharan Africa. Strategies that resulted in improvement in the outcome of interest included financial incentives, increasing the number of providers, home visits, birth plan during antenatal care, cost sharing, task shifting, capacity-building training of providers, mobile health, introduction of a sub-specialty, and supportive supervision. All the studies reported some improvement in quality of care and utilization of primary health care for maternal, new-born and child health. The outcome indicators observed included effective management of malaria in pregnant women and infants, accurate diagnosis of childhood morbidity, timely and appropriate referrals from PHC facilities, effective service delivery for PMTCT of HIV, increase in utilization of PHC facilities for pregnancy care, delivery and postnatal care, increased

Page $10 / 24$ 
male involvement in maternal health, improved capacity of $\mathrm{PHC}$ providers to provide better services, reduction in maternal and child mortality, and reduction in facility-based workload among others.

The successes recorded in the reviewed studies point to the essential role of primary health care in achieving better maternal, new-born and child health outcomes in the region [7-9]. Studies in other countries confirm the important role of primary health care and the place of appropriate interventions in scaling of access and quality of care at this level [42-47]. An appraisal of community health workers prograamme in Malawi, Uganda, and Ethiopia in 2009 showed that effective delivery of care at the primary health level is constrained by issues such as inadequate remuneration which leads to loss of workers from time to time, insufficient attention to quality supervision and continuous training [48]. The results presented in the current review suggest that these constraints among others can be overcome with appropriate design of interventions. Analysis of the effect of applying appropriate interventions in sub-Saharan Africa shows that over 4 million deaths of mothers, newborns, and children will be averted if known effective interventions reach $90 \%$ of targeted populations [49].

Although the reviewed articles reported improvements in primary health care for $\mathrm{MNCH}$, some gaps were identified. For instance, most of the studies did not report on confounders that may have influenced the reported results such as alternative public and private health care outreaches, improvement in economic status, better transportation, and access to health care and environmental factors among others. None of the studies focused specifically on PHC policy. The involvement of government and non-governmental agencies in Ethiopia [9, 10], for instance, strengthened PHC in that country, suggesting the critical role of PHC policy. There is the need for more proactive involvement of governments in enhancing quality and access to $\mathrm{PHC}$ for MNCH care and in reforming $\mathrm{PHC}$ policies to suit prevailing demographic social and economic changes in the country. Results from an analysis of the 2014 PHC reform in Poland suggests the importance of regular revision and scale up of PHC operation and services to reflect changing health needs attuned to current and future demographic structure of a country [50]. Another major recommendation from the case of Poland is the need for the voice of the beneficiaries to be taken into account in policy making and decision-making processes.

None of the studies focussed on maternal mental health, perhaps indicating a paucity of research on mental health and a weak health system attention to mental health in sub-Saharan Africa [51]. Maternal mental health affects child care [52-54]. There is the need to scale up the scope of PHC services to include maternal mental health where it does not exist in sub-Saharan Africa. A study conducted in Australia showed the importance of scaling up the scope of PHC services for maternal health to include maternal mental health four years postpartum[55]. In this Australian study, almost one in three women reported depressive symptoms at least once in the first 4 years after birth. The prevalence of depressive symptoms at 4 years postpartum was $14.5 \%$ and was higher than at any time-point in the first 12 months postpartum. More studies on maternal mental health are needed in sub-Saharan Africa.

Of note in this review is the fact that in spite of the positive results reported in the 25 articles, MNCH indicators in many of the countries have not improved substantially. For instance, at the termination of the MDGs in 2015, no progress was made in reduction of MMR in Malawi, Kenya, Nigeria but Tanzania and Ethiopia were reported to have achieved a level of change that was viewed as progress [3]. This suggests the need to scale up the successful interventions to sustainable dimensions using various novel approaches including knowledge transfer and community ownership. Most interventions in the region are sponsored by development partners from outside Africa. When the sponsorship ends, the programmes inevitably suffer setbacks in many cases, especially when

Page $11 / 24$ 
there is insufficient governmental or community commitment to sustain the gains. Although there were reports of community involvement in the reviewed articles particularly in recruiting community health volunteers, there is need to strengthen sustained community ownership, including leverage private sector support. Primary health care facilities in many countries in sub-Saharan Africa are more likely to be used by the lower socioeconomic groups than the more privileged groups [10] and many communities in the region have high levels of poverty. Thus, communities alone cannot sustain the gains of successful interventions programmes in primary health facilities, and as such political commitments from the governmental sector would be required.

The mhealth strategy is imperative given the increasing use of mobile telecommunication in many countries in sub-Saharan Africa. The mhealth in Malawi reduced the burden of care-seeking in health facilities for childhood illnesses particularly fever [32]. This indicates that mhealth strategy would reduce facility-level workload of PHC workers. Previous studies point to the adverse effect of large workload on providers' efficiency in service delivery [56]. Evidence from the reviewed studies and findings from other regions $[57,58]$ show that mHealth adapted to suit local peculiarities of the served community would increase the quality of care given by primary health care providers, and promote access to facility-based maternity care.

In some countries such as Uganda, Ethiopia, Nigeria, the outcomes of interventions with regard to skilled attendance during delivery was not statistically significant. Yet, it is evident that skilled attendance at delivery is one of the most important components that can substantially improve maternal health and reduce maternal and perinatal mortality in many African countries. This is an indication that more needs to be done with respect to promoting the use of facility delivery. In addition to training more skilled $\mathrm{MNCH}$ providers and strengthening $\mathrm{PHC}$ facilities, there is the need for projects that will improve providers' attitude to clients and reduce long waiting times. Many studies across sub-Saharan Africa point to these as major deterrents to health facility utilization for delivery and maternal, new-born and child care [59-61]. In addition, evidence from studies indicates that there still exists a deep-seated confidence in traditional forms of maternal care in many countries in sub-Saharan Africa [62, 63]. This points to the need for strategies that will involve traditional birth attendants such as the "guide team" in Ethiopia [26] to encourage skilled delivery care.

A limitation of the review is the exclusion of studies published in other languages. In spite of this limitation, this review presents systematic evidence on the effectiveness of PHC in improving maternal, new-born and child health in sub-Saharan Africa.

\section{Policy and programmatic implications}

The results of this systematic review suggest the need for implementation research to determine how to scale up effective interventions for improving women's access to maternal, new-born and child health care at the primary health care level in sub-Saharan African countries. Universal access to maternal health care premised on primary health care to reach the most vulnerable and poor communities hold the key to reducing the current high burden of maternal and child mortality in the region. A knowledge transfer component should always be integrated into any intervention that is designed to improve the delivery of primary maternal health care in Africa so as to ensure that any knowledge gained are transferred into over-arching policies and programs. A strong political will backed by accountability mechanisms through political leadership and ownership would be critical to ensure the adoption and scaling of the best practices in primary maternal health care in the region any time soon. 


\section{Regions where there are few studies}

\section{Conclusions}

In conclusion, the results of this review reveal a large number of existing information in sub-Saharan African countries on innovative practices that can be adopted to increase women's access to primary maternal health care for preventing maternal, new-born and child morbidity and mortality. However, the challenge remains on identifying ways to scale such interventions to reach the most vulnerable citizens for greater impact in all parts of the continent.

\section{Conflict Of Interest}

\section{The authors declare no conflict of interest.}

\section{References}

1. Kinney MV, Kerber KJ, Black RE, Cohen B, Nkrumah F, Coovadia H, et al. Sub-Saharan Africa's mothers, newborns, and children: where and why do they die? PLoS Med. 2010;7:e1000294.

2. World Health Organization. Packages of interventions for family planning, safe abortion care, maternal, newborn and child health. WHO; 2010.

3. World Health Organization. Trends in maternal mortality: 1990 to 2015. Estimates by WHO, UNICEF, UNFPA, The World Bank and the United Nations Population Division. Geneva, Switzerland: World Health Organization; 2015.

4. Black RE, Morris SS, Bryce J. Where and why are 10 million children dying every year? The Lancet. 2003;361:2226-34.

5. World Health Organization. World health statistics 2016: monitoring health for the SDGs, sustainable development goals. Geneva: World Health Organization; 2016.

6. ICF International. The DHS Program STATcompiler. 2015. http://www.statcompiler.com.

7. World Health Organization. Declaration of Alma-Ata: International Conference on Primary Health Care, AlmaAta, USSR, 6-12 September 1978. Retrieved February. 1978;14:2006.

8. World Health Organization. Ouagadougou Declaration on primary health care and health systems in Africa: achieving better health for Africa in the new Millennium. Brazzaville: WHO Regional Office for Africa. 2008.

9. African Union. Draft Policy Brief for the International Conference on Maternal, Newborn and Child Health $(\mathrm{MNCH})$ in Africa. Johannesburg, South Africa. Policy Brief. 2013.

10. Memirie ST, Verguet S, Norheim OF, Levin C, Johansson KA. Inequalities in utilization of maternal and child health services in Ethiopia: the role of primary health care. BMC health services research. 2016;16:1.

11. Hill AG, MacLeod W, Joof D, Gomez P, Walraven G. Decline of mortality in children in rural Gambia: the influence of village-level Primary Health Care. Tropical Medicine \& International Health. 2000;5:107-18.

12. Federal Government of Nigeria. Integrating Primary Health Care Governance in Nigeria (PHC Under One Roof): Implementation manual. National Health Care Development Agency; 2013.

13. NPHCDA. National Primary Health Care Development Agency: Minimum Standards for Primary Health Care in Nigeria. Abuja, Nigeria: Department of Planning, Research and Statistics, National Primary Health Care 
Development Agency; 2012. www.nphcda.gov.ng.

14. Christopher JB, Le May A, Lewin S, Ross DA. Thirty years after Alma-Ata: a systematic review of the impact of community health workers delivering curative interventions against malaria, pneumonia and diarrhoea on child mortality and morbidity in sub-Saharan Africa. Human resources for health. 2011;9:27.

15. Guyatt GH, Oxman AD, Vist GE, Kunz R, Falck-Ytter Y, Alonso-Coello P, et al. GRADE: an emerging consensus on rating quality of evidence and strength of recommendations. BMJ: British Medical Journal. 2008;336:924.

16. Oxman AD, GRADE Working Group. Grading quality of evidence and strength of recommendations. Bmj. 2004;328:1490-4.

17. Mushi D. Impact of cost sharing on utilization of primary health care Services: providers versus household perspectives. Malawi Medical Journal. 2014;26:83-9.

18. Mushi D, Mpembeni R, Jahn A. Effectiveness of community based safe motherhood promoters in improving the utilization of obstetric care. The case of Mtwara Rural District in Tanzania. BMC pregnancy and childbirth. 2010;10:14.

19. Lund S, Rasch V, Hemed M, Boas IM, Said A, Said K, et al. Mobile phone intervention reduces perinatal mortality in Zanzibar: secondary outcomes of a cluster randomized controlled trial. JMIR mHealth and uHealth. 2014;2:e15.

20. Lund S, Nielsen BB, Hemed M, Boas IM, Said A, Said K, et al. Mobile phones improve antenatal care attendance in Zanzibar: a cluster randomized controlled trial. BMC pregnancy and childbirth. 2014;14:29.

21. August F, Pembe AB, Mpembeni R, Axemo P, Darj E. Community health workers can improve male involvement in maternal health: evidence from rural Tanzania. Global health action. 2016;9.

22. Magoma M, Requejo J, Campbell O, Cousens S, Merialdi M, Filippi V. The effectiveness of birth plans in increasing use of skilled care at delivery and postnatal care in rural Tanzania: a cluster randomised trial. Tropical Medicine \& International Health. 2013;18:435-43.

23. Basinga P, Gertler PJ, Binagwaho A, Soucat AL, Sturdy J, Vermeersch CM. Effect on maternal and child health services in Rwanda of payment to primary health-care providers for performance: an impact evaluation. The Lancet. 2011;377:1421-8.

24. Haver J, Brieger W, Zoungrana J, Ansari N, Kagoma J. Experiences engaging community health workers to provide maternal and newborn health services: implementation of four programs. Int J Gynaecol Obstet. 2015;Suppl 2. doi:10.1016/j.ijgo.2015.03.006.

25. Karim AM, Admassu K, Schellenberg J, Alemu H, Getachew N, Ameha A, et al. Effect of Ethiopia's health extension program on maternal and newborn health care practices in 101 rural districts: a dose-response study. PLoS One. 2013;8:e65160.

26. Sibley LM, Tesfaye S, Fekadu Desta B, Hailemichael Frew A, Kebede A, Mohammed H, et al. Improving maternal and newborn health care delivery in rural Amhara and Oromiya regions of Ethiopia through the Maternal and Newborn Health in Ethiopia Partnership. Journal of Midwifery \& Women's Health. 2014;59:S620.

27. Adam MB, Dillmann M, Chen M, Mbugua S, Ndung'u J, Mumbi P, et al. Improving maternal and newborn health: effectiveness of a community health worker program in rural Kenya. PloS one. 2014;9:e104027.

28. Singh D, Negin J, Orach CG, Cumming R. Supportive supervision for volunteers to deliver reproductive health education: a cluster randomized trial. Reproductive Health. 2016;13:126. 
29. Cox S, Mpofu F, Berg A, Rode H. The impact of subspecialty services on health care delivery-a community health centre based study. South African Medical Journal. 2006;96:945-9.

30. Doherty T, Chopra M, Nsibande D, Mngoma D. Improving the coverage of the PMTCT programme through a participatory quality improvement intervention in South Africa. BMC public health. 2009;9:406.

31. Nsibande D, Doherty T, ljumba P, Tomlinson M, Jackson D, Sanders D, et al. Assessment of the uptake of neonatal and young infant referrals by community health workers to public health facilities in an urban informal settlement, KwaZulu-Natal, South Africa. BMC health services research. 2013;13:47-47.

32. Fotso JC, Bellhouse L, Vesel L, Jezman Z. Strengthening the home-to-facility continuum of newborn and child health care through mHealth: Evidence from an intervention in rural Malawi. Etude de la Population Africaine. 2015;29:1663.

33. Robertson L, Mushati P, Eaton JW, Dumba L, Mavise G, Makoni J, et al. Effects of unconditional and conditional cash transfers on child health and development in Zimbabwe: a cluster-randomised trial. The Lancet. 2013;381:1283-92.

34. Okoli U. Strengthening Primary Health Care Services in Rural Nigeria: The Potential of Using Midwives as Skilled Birth Attendants. Health Systems and Policy Research. 2016.

35. Okoli U, Morris L, Oshin A, Pate MA, Aigbe C, Muhammad A. Conditional cash transfer schemes in Nigeria: potential gains for maternal and child health service uptake in a national pilot programme. BMC pregnancy and childbirth. 2014;14:408.

36. Samuel FO, Olaolorun FM, Adeniyi JD. A training intervention on child feeding among primary healthcare workers in Ibadan Municipality. African journal of primary health care \& family medicine. 2016;8:1-6.

37. Ordinioha B, Seiyefa B. Improving skilled attendants at birth: Experience in a primary health care facility in Rivers State, South-South Nigeria. Journal of Community Medicine and Primary Health Care. 2013;25:59-66.

38. Bello DA, Hassan ZI, Afolaranmi TO, Tagurum YO, Chirdan OO, Zoakah Al. Supportive supervision: an effective intervention in achieving high quality malaria case management at primary health care level in Jos, Nigeria. Annals of African Medicine. 2013;12:243-51.

39. Brazier E, Andrzejewski C, Perkins ME, Themmen EM, Knight RJ, Bassane B. Improving poor women's access to maternity care: Findings from a primary care intervention in Burkina Faso. Social science \& medicine. 2009;69:682-90.

40. Suh S, Moreira P, Ly M. Improving quality of reproductive health care in Senegal through formative supervision: results from four districts. Human Resources for Health. 2007;5:26.

41. Pence BW, Nyarko P, Phillips JF, Debpuur C. The Effect of Community Nurses and Health Volunteers on Child Mortality: The Navrongo Community Health and Family Planning Project. Working paper. New York, USA: Population Council; 2005.

42. Fischer EA, Jayana K, Cunningham T, Washington M, Mony P, Bradley J, et al. Nurse mentors to advance quality improvement in primary health centers: lessons from a pilot program in Northern Karnataka, India. Global Health: Science and Practice. 2015;3:660-75.

43. Ghimire M, Pradhan YV, Maskey MK. Community-based interventions for diarrhoeal diseases and acute respiratory infections in Nepal. Bulletin of the World Health Organization. 2010;88:216-21.

44. Macinko J, Guanais FC, De Souza MDFM. Evaluation of the impact of the Family Health Program on infant mortality in Brazil, 1990-2002. Journal of epidemiology and community health. 2006;60:13-9. 
45. Setiawan A, Dignam D, Waters C, Dawson A. Improving access to child health care in Indonesia through community case management. Maternal and child health journal. 2016;20:2254-60.

46. Shei A, Costa F, Reis MG, Ko Al. The impact of Brazil's Bolsa Família conditional cash transfer program on children's health care utilization and health outcomes. BMC international health and human rights. 2014;14:10.

47. Soofi S, Ahmed S, Fox MP, MacLeod WB, Thea DM, Qazi SA, et al. Effectiveness of community case management of severe pneumonia with oral amoxicillin in children aged 2-59 months in Matiari district, rural Pakistan: a cluster-randomised controlled trial. The Lancet. 2012;379:729-37.

48. Hermann K, Van Damme W, Pariyo GW, Schouten E, Assefa Y, Cirera A, et al. Community health workers for ART in sub-Saharan Africa: learning from experience-capitalizing on new opportunities. Human resources for health. 2009;7:31.

49. Friberg IK, Kinney MV, Lawn JE, Kerber KJ, Odubanjo MO, Bergh A-M, et al. Sub-Saharan Africa's mothers, newborns, and children: how many lives could be saved with targeted health interventions? PLoS Med. 2010;7:e1000295.

50. Mokrzycka A, Kowalska-Bobko I, Sagan A, Włodarczyk WC. The 2014 primary health care reform in Poland: Short-term fixes instead of a long-term strategy. Health Policy. 2016;120:1226-32.

51. Jenkins R, Othieno C, Okeyo S, Aruwa J, Kingora J, Jenkins B. Health system challenges to integration of mental health delivery in primary care in Kenya - perspectives of primary care health workers. BMC Health Services Research. 13.

52. Harpham T, Huttly S, De Silva MJ, Abramsky T. Maternal mental health and child nutritional status in four developing countries. Journal of epidemiology and community health. 2005;59:1060-4.

53. Koutra K, Chatzi L, Bagkeris M, Vassilaki M, Bitsios P, Kogevinas M. Antenatal and postnatal maternal mental health as determinants of infant neurodevelopment at 18 months of age in a mother-child cohort (Rhea Study) in Crete, Greece. Social psychiatry and psychiatric epidemiology. 2013;48:1335-45.

54. Patel V, Rahman A, Jacob K, Hughes M. Effect of maternal mental health on infant growth in low income countries: new evidence from South Asia. BMJ: British Medical Journal. 2004;328:820.

55. Woolhouse H, Gartland D, Mensah F, Brown S. Maternal depression from early pregnancy to 4 years postpartum in a prospective pregnancy cohort study: implications for primary health care. BJOG: An International Journal of Obstetrics \& Gynaecology. 2015;122:312-21.

56. Ogu RN, Ntoimo LFC, Okonofua FE. Perceptions of women on workloads in health facilities and its effect on maternal health care: A multi-site qualitative study in Nigeria. Midwifery. 2017;55:1-6.

57. Tamrat T, Kachnowski S. Special delivery: an analysis of mHealth in maternal and newborn health programs and their outcomes around the world. Maternal and child health journal. 2012;16:1092-101.

58. Watterson JL, Walsh J, Madeka I. Using mHealth to improve usage of antenatal care, postnatal care, and immunization: A systematic review of the literature. BioMed research international. 2015;2015.

59. Balde MD, Bangoura A, Sall O, Soumah AM, Vogel JP, Bohren MA. Perceptions and experiences of the mistreatment of women during childbirth in health facilities in Guinea: a qualitative study with women and service providers. Reproductive Health. 2017;14:3.

60. Bamidele AR, Hoque ME, Van der Heever H. Patient satisfaction with the quality of care in a primary health care setting in Botswana. South African Family Practice. 2011;53:170-5. 
61. Bohren MA, Vogel JP, Tunçalp Ö, Fawole B, Titiloye MA, Olutayo AO, et al. Mistreatment of women during childbirth in Abuja, Nigeria: a qualitative study on perceptions and experiences of women and healthcare providers. Reproductive Health. 2017;14:9.

62. Sialubanje C, Massar K, Hamer DH, Ruiter RA. Reasons for home delivery and use of traditional birth attendants in rural Zambia: a qualitative study. BMC pregnancy and childbirth. 2015;15:216.

63. Doctor HV, Dahiru T. Utilization of non-skilled birth attendants in Northern Nigeria: a rough terrain to the health-related MDGs. African Journal of Reproductive Health. 2010;14:37-45.

\section{Tables}

Table 1: Summary of the reviewed studies 


\begin{tabular}{|c|c|c|c|c|c|}
\hline Authors/Year/Setting & $\begin{array}{l}\text { Study } \\
\text { Design/Objective }\end{array}$ & $\begin{array}{l}\text { Exposure/Follow- } \\
\text { up }\end{array}$ & Intervention & Key findings & $\begin{array}{l}\text { Quality } \\
\text { assessment }\end{array}$ \\
\hline $\begin{array}{l}\text { (1) Basinga et. al. } \\
\text { (2011) } \\
\text { Rwanda }\end{array}$ & $\begin{array}{l}\text { Design: Prospective } \\
\text { study/Experimental } \\
\text { design } \\
\text { Objective: To access } \\
\text { the effect of an } \\
\text { incentive-based bonus }\end{array}$ & $\begin{array}{l}\text { Baseline: June- } \\
\text { October, } 2006 \\
\text { End-line survey: } \\
25 \text { months after }\end{array}$ & $\begin{array}{l}\text { Financial } \\
\text { incentive: } \\
\text { Payment for } \\
\text { performance } \\
(\mathrm{P} 4 \mathrm{P})\end{array}$ & $\begin{array}{l}\text { Increase in } \\
\text { antenatal care } \\
\text { quality, the number } \\
\text { of institutional } \\
\text { deliveries and. } \\
\text { preventive care } \\
\text { visits by children, . } \\
\text { No improvements } \\
\text { in the number of } \\
\text { women completing } \\
\text { four antenatal care } \\
\text { visits and children } \\
\text { receiving full }\end{array}$ & High \\
\hline
\end{tabular}

(2) Okoli, U. (2016) Design: Evaluation Nigeria

Research/baseline

immunisation. and end line survey Objective: To assess the potential of using midwives as skilled birth attendants in providing access to maternal health services in rural Nigeria

$\begin{array}{ll}\text { Baseline } & \text { Increase in } \\ \text { November } & \text { number of } \\ \text { 2012.Follow-up in } & \text { facility-based } \\ \text { August 2014 } & \text { providers }\end{array}$

August $2014 \quad$ providers

\section{(3) Haver et al. (2015)}

Rwanda/Nigeria

\begin{tabular}{|c|c|c|}
\hline $\begin{array}{l}\text { Design: Case/control } \\
\text { studies } \\
\text { Objective: To review } \\
\text { implementation of } \\
\text { jhpiego's programs } \\
\text { on engaging } \\
\text { community health } \\
\text { workers to provide } \\
\text { maternal and new- } \\
\text { born health services. }\end{array}$ & $\begin{array}{l}\text { Rwanda: 2010- } \\
2011\end{array}$ & $\begin{array}{l}\text { Rwanda: Task- } \\
\text { shifting } \\
\text { Nigeria - } \\
\text { implementation } \\
\text { of community- } \\
\text { directed } \\
\text { intervention } \\
\text { (CDI) to } \\
\text { address } \\
\text { malaria in } \\
\text { pregnancy in } \\
\text { Akwa Ibom } \\
\text { state, Nigeria. }\end{array}$ \\
\hline
\end{tabular}

Increase in new High

ANC visits, focused

antenatal care

attendance,births

by skilled birth

attendants ,

postnatal visits

and the number of

women using

contraceptive

methods.

Rwanda: Increase Medium

in the number of

pregnant women

accompanied to the

health centre for

care and delivery.

Nigeria:

Significant

increase in the

coverage of IPTp

and insecticide-

treated nets and

prenatal care

attendanceIncrease

in the proportion of

pregnant women

taking at least two

sulfadoxine-

pyrimethamine

doses during

pregnancy.

(4) Adam, M.B. et al. Design: Quasi-

(2014)

Kenya
Medium

\author{
experimental non- \\ equivalent \\ comparison group \\ design \\ Objective: To \\ describe the \\ effectiveness of a \\ volunteer community \\ health worker \\ project.
}

2009-2011

Recruitment of community

health

volunteers

Intervention was exposure to the CHW

delivered health messages
Increase in $\mathrm{MNCH}$ knowledge and the number of women delivering under skilled attendance..
(5) Okoli et al., (2014)

Nigeria
Evaluation

Research/Prospective 2012-2014 study
Financial

incentive:
Increase in the monthly average
High 
Objective: To

examine use of a

Conditional Cash

Transfer (CCT)

programme to

encourage use of

critical MNCH

services among rural

women in Nigeria.

(6) Samuel, F.O. et

al (2016)

Nigeria
Conditional

cash transfer

cash transfer

number of women

attending focused 4

or more ANC visits, number of women

receiving two or

more Tetanus

toxoid doses during

pregnancy.
Design: A before and after clinic-based intervention study.

Objective: To

evaluate the effect of

training on the

knowledge, attitudes

and provision of

infant and young child

feeding (IYCF)

information.

\section{Data collected at}

Baseline-after intervention same day \& 4 weeks after $\begin{array}{lll}\begin{array}{l}\text { A 2-day training } \\ \text { intervention }\end{array} & \begin{array}{l}\text { Improvement in } \\ \text { health workers' }\end{array} & \text { Medium } \\ \text { implemented in } & \begin{array}{l}\text { knowledge of } \\ \text { PHC facilities } \\ \text { IYCFattitudes and } \\ \text { practices of one-on- }\end{array} & \\ \text { using } & \text { one individualized } \\ \text { lectures, } & \text { infant and young } \\ \text { interactive } & \text { child feeding } \\ \text { sessions, group } & \text { counseling on } \\ \text { work, quizzes, } & \text { congs and role } & \\ \text { song to provide } & \\ \text { playformation and } & \\ \text { training on } & \\ \text { current global } & \\ \text { Infant and } & \\ \text { Young Child } & \\ \text { Feeding (IYCF). } & \end{array}$

(7) Fotso et al., (2015)

Malawi
July 2011 and June 2013
A two-arm quasiexperimental, prepost design.
Mhealth: Chipatala cha pa Foni (CCPF) - or Health Centre by Phone.
Adjusted effects of High the intervention shows a large, positive effect on the aggregate home-based care for child health.
Objective: To assesses the impact of a mobile health (mHealth) intervention on uptake of home-based care for new-born and child health, and investigates the extent to which uptake of home-based care resulted in lessened pressure on health facilities for conditions that can be handled at the household level.

(8) Robertson et al., (2013)

Zimbabwe
Design: A matched cluster-randomised controlled trial

\section{9-2011}

(1)

Financial
incentive:
Unconditional
and conditional
cash transfers

\author{
Increase in the High \\ proportion of \\ children aged 0-4 \\ years with birth \\ certificates, and \\ complete \\ vaccination records
}

Objective: To

investigate the

effects of

unconditional cash

transfers (UCTs) and 
conditional cash

transfers (CCTs) on

birth registration,

vaccination uptake,

and school

attendance in

children in Zimbabwe.

(9) Ordinioha \&

Seiyefa (2013)

Nigeria
Design: Before-and-

after design

Objective: To

highlight the

experience of a $\mathrm{PHC}$

facility in south-south

Nigeria, in

encouraging the

utilization of its

maternity service
(10) Mushi (2014)

Tanzania
Design: Evaluation

study (survey)

Objective: To assess

the impact of a

community health

fund (CHF), a cost-

sharing programme

on quality of care,

attendance and

utilization of PHC

services as the $\mathrm{CHF}$

improves.
A community

education for

traditional

rulers, women

groups,

religious

organisations

and traditional

birth

attendants..

Implementation

of upfront

collection of a

delivery fee

equivalent to

the average

charges by

TBAs and less

than the fee for

normal delivery

in a teaching

hospital.
Antenatal

registration and

ANC-Delivery ratio

increased.
Medium

Cost-Sharing

Medium

primary health care

improved.

Attendance by all

categories of

clients for $\mathrm{MCH}$

care almost

doubled andunder-5

mortality declined

in one district.

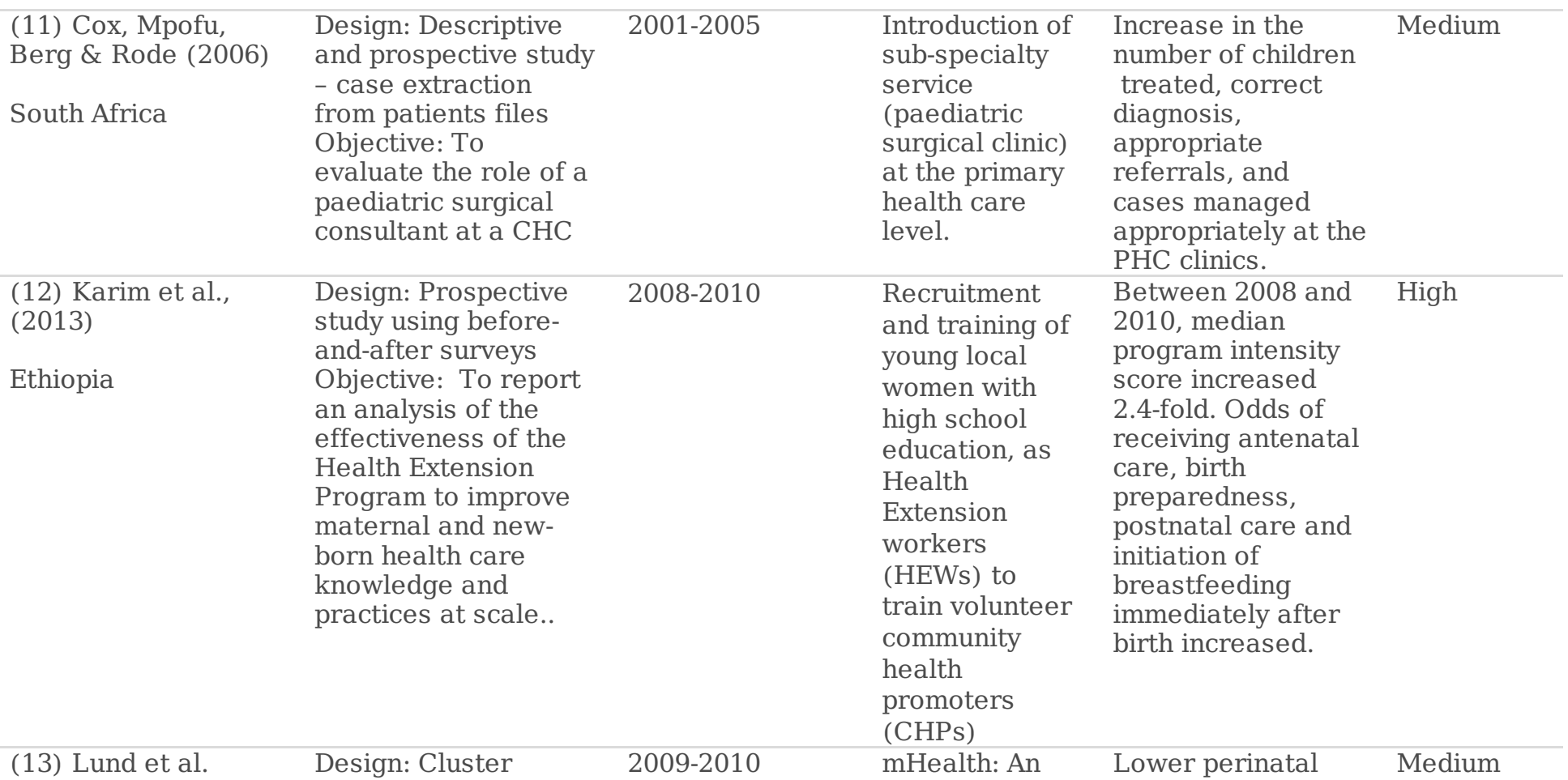


(2014)

Zanzibar,Tanzania

(14) Lund et al

(2014)

Zanzibar,Tanzania randomized,

controlled trial

Objective: To

evaluate the

association between

a mobile phone

intervention and

perinatal mortality in

a resource-limited

setting.

Design: cluster-

randomized

controlled trial

Objective: To

evaluate the

association between

a mobile phone

intervention "wired

mothers" and

antenatal care in

Zanzibar.

(15) Mushi et al.

(2010)

Tanzania

mHealth: Same
as above

as above

automated

short message

service (SMS)

system

mortality in the

intervention sites,

reduction in in

stillbirth and death

within the first 42

days of life.

Increase in High

antenatal care

attendance and

improved timing

and quality of

antenatal care

services.
Design: Pre-post

comparison of the

same group

Objective: To

describe and analyse

the process and the

effectiveness of a

community-based

intervention package

for Safe Motherhood
2004-20

Design: A quasi-

experimental study

(non-equivalent

group)

Objective: To

evaluate the effect of

HBLSS training in the

community on male

involvement in

maternal health in a

rural area

Training of
safe
motherhood
promoters
(SMP), home
visits, to
educate
pregnant
women and
their husbands
and key
community
members,
follow-up of
pregnant
mothers.

2012-2014

Health workers

trained to use

Home Based

Life-Saving

Skills by

community

health workers.

(HBLSS).

The health

workers then

trained

community

health workers

(CHWs)

(17) Sibley et al.,

(2014)

Ethiopia

\author{
Design: Uncontrolled \\ before/after study \\ design (surveys) \\ Objective: To \\ describe the extent to \\ which the Maternal \\ Health in Ethiopia \\ Partnership \\ (MaNHEP) project's \\ objectives were met
}

Baseline survey
June-Sept 2010
End line May-
August 2012

1) $\mathrm{A}$

Community

Maternal and

New-born

Health

(CMNH)

training

program 2)

Continuous

quality

improvement

through Quality

improvement

teams 3)

Behaviour

change

communication
The proportion of

men accompanying

their wives to

antenatal and

delivery improved.

Shared decisionmaking for place of

delivery improved

markedly.

Deliveries with

High

and early ANC

booking increased

significantly.

\section{High}

(2)

f

( 
Improved perinatal

survival.

(18) Doherty et al (2009)

South Africa

\author{
Design: Descriptive \\ Objective: To present \\ results of a \\ participatory \\ intervention to \\ improve an integrated \\ PMTCT programme \\ in a rural district in \\ South Africa \\ 2007-2008
}

(19) Brazier et al. (2009)

Burkina Faso
Design: Baseline and 2003-2006 endline population-

based surveys Objective: To present results of a threeyear maternal health intervention aimed at influencing access to skilled maternity care during childbirth intervention

included improving the quality, availability, and accessibility of routine and emergency obstetric care (EMOC). Communitylevel interventions focused on increasing women's information about services.

(20) Bello et al (2016)

Nigeria
Design: quasiexperimental Case-control study Objective: To measure the effect of supportive supervision of $\mathrm{PHC}$ workers in malaria case management for under-5 children.

12 weeks

Local

Government supervisors were trained on supportive supervision. They carried out supportive supervision in the intervention sites while the traditional supervisory method continued in the control sites.

(21) Suh, Moreira \& Ly (2007)

Senegal
Design: Evaluation study Objective: To assess how formative supervision affected service quality and community involvement in improving quality of service

Design: Pair-matched

2014-2015 cluster randomized trial
2003-2005

A program of formative supervision which uses observation emphasizing problemsolving approach
Coverage of CD4 testing increased from 40 to $97 \%$, uptake of maternal nevirapine from 57 to $96 \%$, uptake of infant nevirapine from 15 to $68 \%$ and six week PCR testing from 24 to $68 \%$.

A large increase in High the per cent of births in health facilities in the intervention district; increase in delivery by skilled attendant compared to the comparison district.

High
Medium

Significant increase Medium
in knowledge of
malaria, referral to
a secondary
facility, correct
adherence to
guidelines, eliciting
of symptoms of
malaria from a sick
child, and
treatment and
dosing of patients.

(22) Singh et al

(2016)

Uganda
Community

Health workers were recruited and trained to supervise the

Improvement in
technical
competence in
infection
prevention, skills in
family planning
consultation,
management of
staff and services,
record-keeping,
and community
involvement.
More functioning
tippy taps. in the
intervention
villages than in
control villages..


Objective: To

determine if

supportive

supervision would

improve retention

rates, numbers of

home visits related to

pregnant women and

new-born babies, and

improve specific

outcomes related to

hygiene.

(23) Pence et al., 2005

Ghana
Community

Health

Volunteers
Visits to pregnant women by CHVs increased. Visits to new-borns improved.
(24) Nsibande et al 2013

South Africa
(25) Magoma et al 2013

Tanzania
Design: Community-

randomized

controlled experiment line 2000

Objective: To

examine the impact of

Community Health

and Family Planning

Project (CHFP) on

under-five mortality.

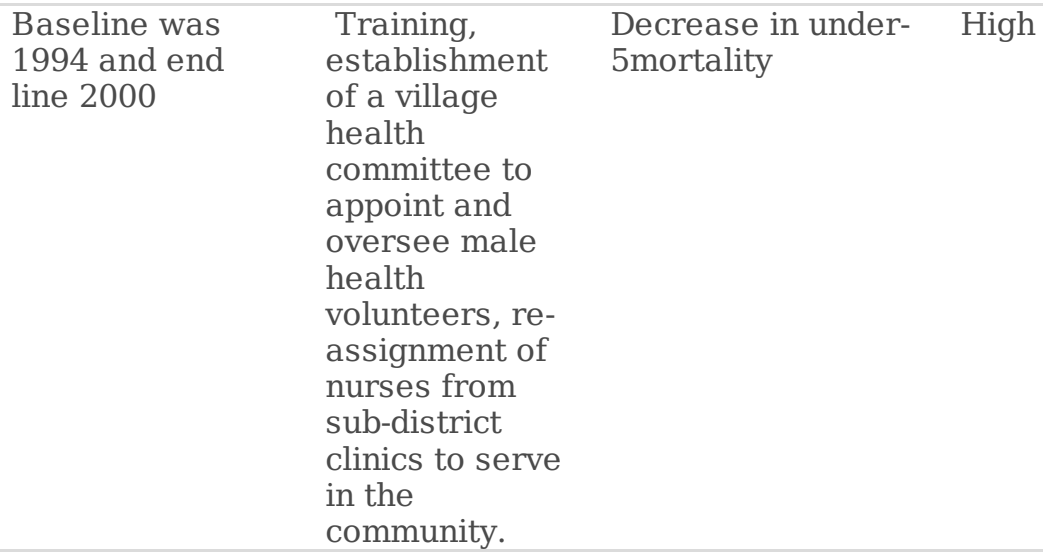
1994 and end

Training, establishment of a village health committee to appoint and oversee male health volunteers, reassignment of nurses from sub-district clinics to serve in the community.

Decrease in under- High 5mortality

Design: Cluster-

randomised

controlled trial

Objective: To

develop, evaluate and

cost an integrated

and scalable home

visit package

delivered by CHWs.
2008-2011

CHWs visited mothers and new-borns during the last trimester of pregnancy and during the early postnatal period to deliver a package relating to child health.

Introduction and promotion of birth plans during ANC to prepare women and their families for birth and complication readiness. maternal and

Increase in High

referrals and high compliance with CHW

High

Skilled delivery and High postnatal care in the first month of delivery was higher in the intervention units than in the control

postnatal care of skilled care at delivery and for

Dec 2009

Objective: To

effectiveness of birth

plan in increasing use

\section{Figures}




\section{Selection of Study}

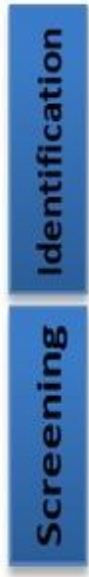

\section{DATABASES: Record identified}

through database search $(n=1594)$
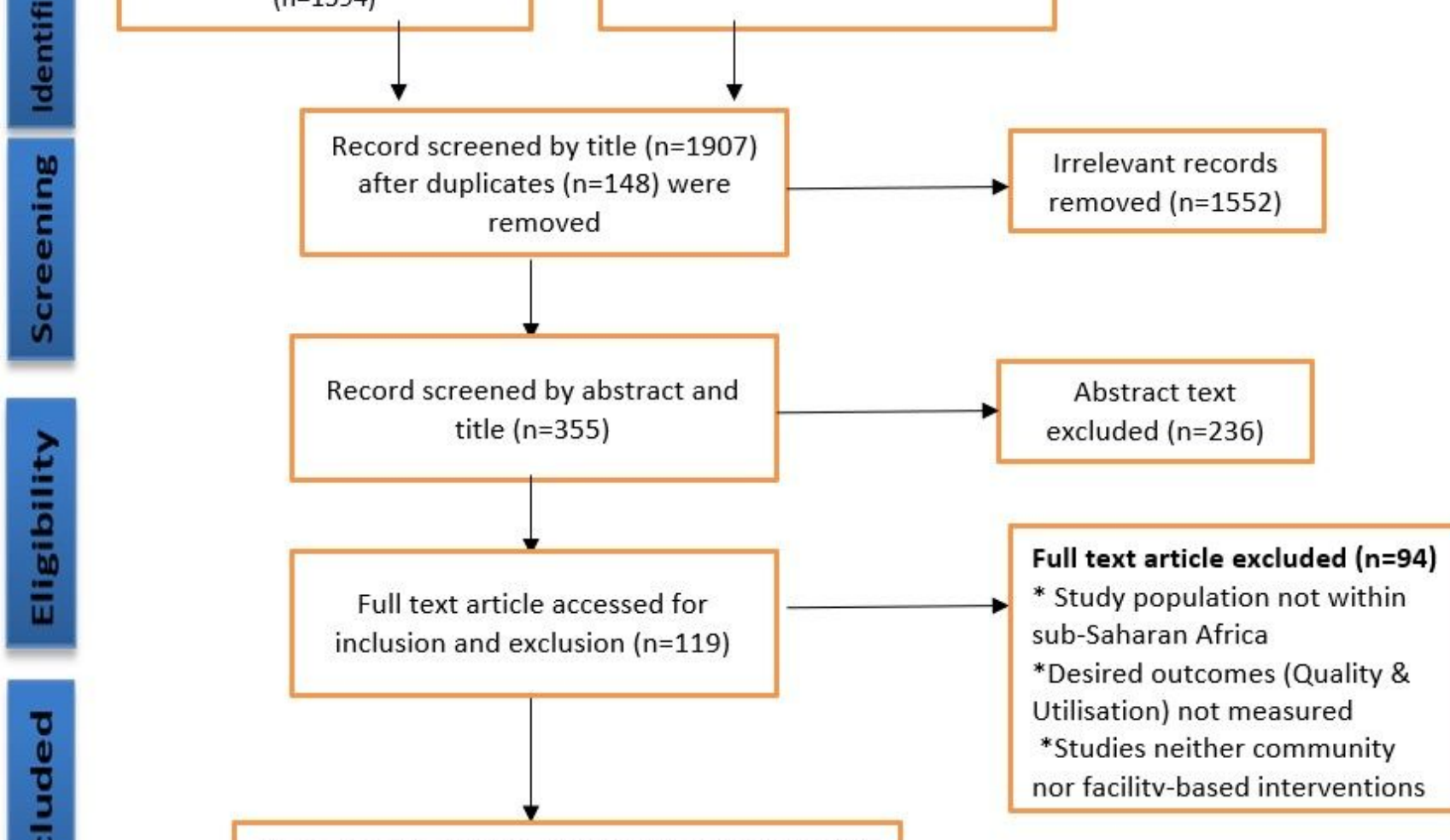

Article included in the systematic review $(n=25)$

Fig 1: Flow diagram of study selection according to PRISMA Flow Chart

\section{Figure 1}

Flow diagram of study selection according to PRISMA Flow Chart 